\title{
Neuropsychobiology 1989;21:224
}

\section{Subject Index}

\section{ACTH 192}

Adjective checklist 197 Adolescent 9 ß-Adrenoreceptor sensitivity

177 Aggression 9 Alprazolam 182 Aminooxyacetic acid 152 Amphetamine 117 Anorexia nervosa 22, 187 Antiepileptic drugs 157 Anxiety 197 - disorder 124

Anxiolysis 197

Arousal 170

Attention 31,90

Auditory evoked potential amplitude 48 Autism 48

Benzodiazepine receptors 14 Brain $\alpha$ i-receptors 100

- mapping 170

Bulimia nervosa 187

Buspirone 124

Calcium 67 Carbohydrates 84 Cerebral atrophy 187

- blood flow 117 Cerebrospinal fluid 182 Children 43 Cholecystokinin 22 Cholinergic drug 94

- system 31, 76 Clomipramine 111 Clonidine 205

Cognitive component Ni 146

- performance 131, 187

Common cold 90

Correlation analysis 43

Corticotropin-releasing factor 22

Cortisol 1,71, 111, 192

Craniopharyngioma 17 
Depression 1,71, 109, 177, 192 Deproteinized sera 14 Desipramine 111,177 Dopamine 37

Dopaminergic metabolism 48 Dose-response study 197 Drowsiness 170 Drug evaluation 124

\section{EEG power spectrum 157}

- sleep 100

Electroencephalogram 104,216 Electroencephalography 205 Endogenous modulators 14 ß-Endorphins 192 Epidemiology 109 Erythrocytes 53

Eserine 205

Ethanolamine-O-sulfate 152 Euphoric response 1 Event-related potentials 59, 146 Evoked responses 157

F|waves 104 Fenfluramine 48, 192 Fentanyl 1 Fluspirilene 197 Food-seeking behavior 17 Forced swimming test 152

GABA transaminase inhibitors

152 Genetic determination 111 Growth hormone 1,111

Haloperidol 37, 131,205 Hamilton Anxiety Rating Scale 124

- Scale 197 Healthy conditions 14

- subjects 37 Hyperphagia 17 Hypertension 84 Hypothalamic damage 17 
Imipramine 182 Influenza 90

Information processing 146 Insomnia 109 Isoproterenol 177

Lamotrigine 157 Long loop reflexes 104

Man 31, 76

Membrane transport 53

Memory 76

Monoamine metabolites 182

Mood performance 84

Multi-infarct dementia 141

Neuroleptic-induced extrapyra-

midal symptoms 67 Neuropeptide(s) 22, 182 Nicotine 216

Nocturnal penile tumescence 71 Nootropic(s) 94,141 Noradrenaline 1 Normal subjects 43

Opiate receptor 1 Oxiracetam 141

P300 59, 94

Panic disorder 182

- syndrome 197

Paranoid versus nonparanoid 59

Pattern-reversal visual evoked

potentials 43 Performance $90 \mathrm{pH} 67$

Pharmaco-EEG 157 Phenytoin 157 Piracetam 205 Prazosin 100 Primary degenerative dementia

141 Prolactin 1,71, 111,192 Protein 67 
Psychiatric disorders 14 Psychological models 31 Pyridoxine 104

Rat 100

Reaction time 37, 90 Receptor binding ex vivo 100 Remoxipride 131 Retrieval 76

Schizophrenia 59, 117, 146

Schizophrenic patients 131

Scopolamine 31,76

Selective attention impairment 146

Sensorimotor performance 131

Serotonin 9, 22, 84

Single-trial potential variability 48

Sleep 84

- EEG 71

Smoking 216

Somatosensory evoked potentials 104

Spatial attention 94

Suicide 9

Sulfhydryl groups 53

Theophylline 104 Time perception 37 Trimipramine 71 Tryptophan 84 L-Tryptophan 53, 170 Twins 43 L-Tyrosine 53

Valproicacid $152 \gamma$-VinylGABA 152 Visual event-related brain potentials 94

Working memory 31

Yohimbine 205 